\title{
Analyse de la conception d'un montage d'usinage à l'aide d'éléments modulaires
}

\author{
SAID Zirmi ${ }^{1}$, Henri PARIS ${ }^{1, a}$ et Idir Belaidi ${ }^{2}$ \\ 1 Laboratoire G-SCOP, BP 53, 38041 Grenoble Cedex 9, France \\ 2 Laboratoire de productique, Université de Boumerdes, 35000 Boumerdes, Algérie
}

Reçu le 27 janvier 2006, accepté le 7 mars 2007

\begin{abstract}
Résumé - Un montage d'usinage est un outillage indispensable pour fixer la pièce solidement à la bonne position dans l'espace de travail de la machine outil. La conception du montage d'usinage joue un rôle important pour obtenir une pièce usinée de bonne qualité. Nous avons déjà formalisé les connaissances sur les prises de pièce de manière à pouvoir les gérer tout au long du processus de conception de la gamme d'usinage. Maintenant nous nous intéressons à la conception du montage d'usinage de manière à passer du modèle prise de pièce retenu à la solution technologique qui sera utilisée sur le poste de travail. Cet article aborde le problème de conception du montage d'usinage modulaire du point de vue technologique dans sa globalité ; c'est-à-dire le choix et le placement des éléments technologiques en tenant compte de toutes les contraintes de qualité, d'accessibilité et de comportement mécanique du système pièce-montage d'usinage-outil de coupe durant l'usinage.
\end{abstract}

Mots clés : Conception / montage d'usinage / accessibilité / qualité

Abstract - Analysis of modular fixtures design. A fixture is essential equipment to firmly fix the part at the good position in the workspace of the machine tool. The design of the fixture plays an important part to obtain a machined part of good quality. We already formalized knowledge on the fixturing so as to be able to manage them throughout process planning design. Now we are interested in the design of the fixture so as to pass from the fixturing model to the technological solution which will be used on the machine tool. This paper deals with the problem of modular fixture design with a technological point of view. The choice and the placement of element are carried out in taking account of all the constraints: quality, accessibility and mechanical behaviour of the system part fixture cutting tool during the machining.

Key words: Design / fixture / accessibility / optimization

\section{Introduction}

La conception et la réalisation du montage d'usinage sont des étapes importantes dans la maîtrise de la qualité des pièces usinées. Depuis quelques années, la littérature propose de nombreuses études sur la conception des montages d'usinage pour le fraisage. Ces travaux concernent la modélisation des connaissances pour l'aide à la conception des montages d'usinage sous forme d'une base de règles [1] et la conception automatique de montages modulaires pour les pièces prismatiques [2]. Ces travaux considèrent plus particulièrement l'accessibilité des différentes surfaces de la pièce pour choisir les surfaces de posage [3], la conception du bridage et l'optimisation des efforts de serrage en tenant compte de la

\footnotetext{
a Auteur correspondant : henri.paris@hmg.inpg.fr
}

stabilité et de l'équilibre de la pièce [4-8], la conception de montages d'usinage assistée par ordinateur en proposant différentes approches [9]; géométriques [10], ou à l'aide d'algorithmes génétiques [11]. D'autres travaux s'intéressent à l'analyse de l'erreur de positionnement de la pièce sur ses appuis [12] et à la gestion des collisions entre les éléments constituant le montage et les outils de coupe $[13,14]$. La validation de la qualité de positionnement est abordée de manière à s'assurer du bon positionnement des surfaces usinées [15]. Des modèles plus fins intégrant les phénomènes de frottement sont aussi abordés [16].

Tous ces travaux apportent des solutions à de nombreux sous-problèmes contribuant à enrichir les différents modèles nécessaires pour modéliser le comportement des montages d'usinage et pour apporter des éléments au processus de conception de ces montages d'usinage. 
Le problème qui nous intéresse ici est un problème prenant la conception des montages d'usinage modulaires dans sa globalité et répondant ainsi à l'ensemble des fonctions attendues. Il s'agit donc d'une optimisation globale. Nous nous intéressons à comprendre comment à partir d'un posage donné, les experts en usinage choisissent les composants technologiques et les relient entre eux et avec la plaque de base.

Notre travail consiste à prendre en considération les différentes contraintes liées à la conception du montage d'usinage modulaire pour le fraisage à savoir le respect de la qualité de la pièce à usiner, l'accessibilité des outils de coupe aux différentes surfaces à usiner et le comportement mécanique de la pièce compatible avec la stabilité et la gestion des déformations locales ou globales de la pièce.

\section{Définition et fonction du montage d'usinage}

\subsection{Qu'est-ce qu'un montage d'usinage?}

Le montage d'usinage est un dispositif permettant de lier mécaniquement la pièce à la table de la machine-outil pendant une ou plusieurs opérations d'usinage. La figure 1 représente un montage d'usinage modulaire. Du point de vue fonctionnel : un montage d'usinage doit assurer essentiellement deux fonctions principales qui sont le positionnement et le maintien de la pièce. Ces fonctions doivent être assurées tout en respectant les contraintes suivantes :

- garantir un libre accès des outils de coupe aux surfaces à usiner,

- garantir un montage et un démontage aisé de la pièce,

- permettre l'évacuation des copeaux et du fluide de coupe,

- garantir la sécurité de l'opérateur pour la mise en œuvre du montage.

\subsection{Fonction d'un montage d'usinage}

Les fonctions principales que doit remplir le montage d'usinage sont :

- Positionner la pièce. Durant l'usinage, l'ensemble des pièces de la série doit être positionné précisément dans l'espace de travail de la machine-outil. Cette condition est nécessaire pour garantir un bon positionnement des usinages sur la pièce et conduit au concept d'isostatisme, de précision et de stabilité. Pour cela, le posage est une combinaison d'appui prépondérant, secondaire et tertiaire qui peut être de type $321 \mathrm{R}$ (plan, linéaire, butée), de type $321 \mathrm{C}$ (plan, centreur, locating) ou de type 411 (pivot, butée, butée) (Fig. 2).

- Maintenir la pièce. Durant l'usinage, la pièce doit être maintenue en position sur ses appuis malgré les sollicitations mécaniques extérieures comme les efforts de coupe, le poids de la pièce et les forces d'inertie.

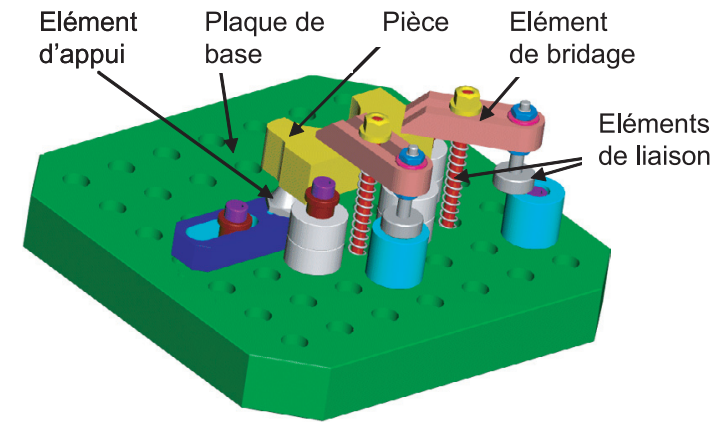

Fig. 1. Exemple d'un montage d'usinage modulaire.

Ce maintien est assuré par un bridage qui peut être de mode bride lorsque l'effort de bridage est opposé à l'appui plan prépondérant, de mode étau lorsque l'effort de bridage est opposé à l'appui linéaire d'orientation et de mode mandrin lorsque la géométrie d'appui participe aussi au bridage (Fig. 3).

- Soutenir la pièce. Dans certains cas, il est nécessaire d'ajouter des soutiens pour éviter les déformations et/ou les vibrations indésirables de la pièce durant son usinage. Le soutien est assuré par des éléments réglables afin de ne pas perturber la qualité de la mise en position.

L'objectif premier du montage d'usinage est de situer précisément la pièce dans l'espace de travail de la machine outil par rapport à un référentiel afin d'assurer l'usinage des entités d'usinage au bon endroit sur la pièce. Cette mise en position ne doit pas être perturbée par les efforts de coupe, de bridage et d'inertie. De plus, les outils de coupe ne doivent pas entrer en collision avec les éléments du montage d'usinage tout au long de leurs trajectoires.

L'ensemble des fonctions du montage d'usinage doit donc répondre à trois contraintes qui sont :

- Contrainte de qualité. L'ensemble des pièces de la série doit occuper la même position dans l'espace de travail. Cette position est perturbée par des défauts issus de la qualité des surfaces appartenant à la pièce assurant un appui et de la qualité du contact entre les éléments technologiques du montage d'usinage et la pièce. Les défauts de positionnement de la pièce doivent donc être compatibles avec la qualité de positionnement des usinages sur la pièce.

- Contrainte d'accessibilité. Les usinages sont réalisés de manière séquentielle avec des outils différents. Les trajectoires sont définies de manière à éviter les collisions entre les outils de coupe et le montage d'usinage. Le système de bridage qui est généralement proche de l'espace de travail des outils de coupe est défini et placé de manière à permettre le passage des outils de coupe. De plus, les éléments technologiques du montage d'usinage sont définis et placés de manière à éviter les collisions entre ces éléments entre eux et avec la pièce.

- Contrainte liée au comportement mécanique. Durant l'usinage, la pièce est soumise aux efforts de coupe, d'inertie, de bridage et de la réaction des appuis. Les 

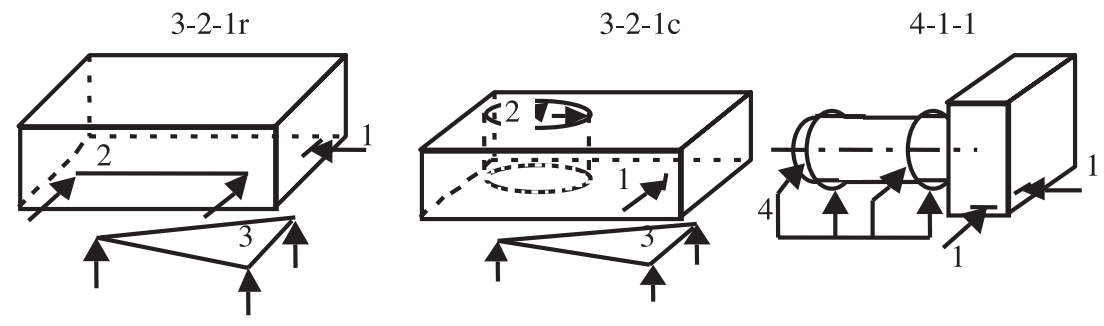

Fig. 2. Les différents types de posage.
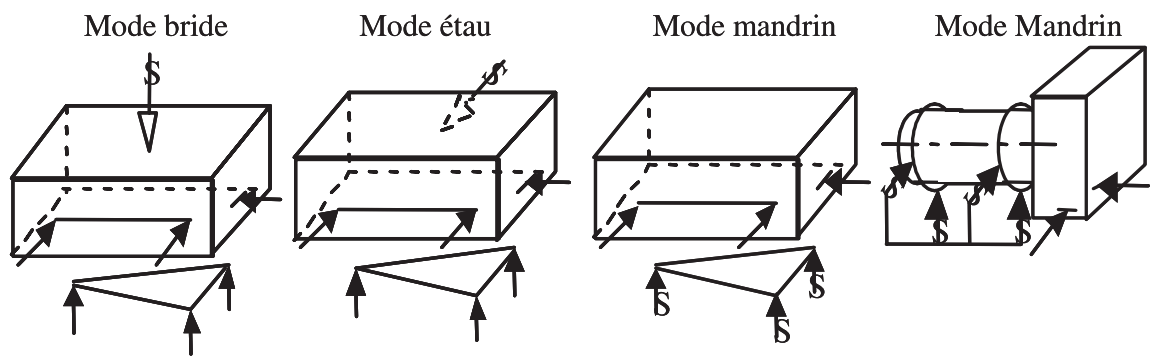

Fig. 3. Les différents modes de bridage.

efforts de coupe sont d'intensités et de directions variables. L'équilibre de la pièce doit être garanti pendant toute la durée de l'usinage. De plus, ces efforts risquent de déformer globalement et localement (au droit des points d'appui et de bridage) la pièce. Les éléments technologiques constituant le montage d'usinage sont donc choisis et placés de manière à garantir la stabilité de la pièce durant l'usinage et de maîtriser les déformations de manière à respecter la qualité demandée.

\section{Domaine d'étude et démarche suivie}

L'objectif principal de nos travaux est d'établir un système de conception du montage d'usinage modulaire pour le fraisage dans un environnement CFAO. Il s'agit de passer d'un modèle entité de prise de pièce [17] à un montage d'usinage. La reconnaissance des entités de prise de pièce dans un environnement CFAO a été abordée lors de travaux précédents au laboratoire. De plus, nous avons mis en place des indicateurs permettant de caractériser les performances des prises de pièce durant le raisonnement de conception de gammes d'usinage [18]. Il s'agit maintenant de matérialiser le montage d'usinage. Pour cela nous disposons des informations suivantes:

- La cinématique de la machine outil. Il s'agit du nombre d'axes et de l'orientation de la plaque de base sur la table de la machine. Nous retenons deux configurations : soit le montage est fixé directement sur la table de la machine outil, soit il est monté sur une équerre ou un cube.

- La liste des opérations d'usinage. Il s'agit d'une suite chronologique d'opérations d'usinage. Chaque opération est typée (ébauche, 1/2 finition et finition). De plus, la surépaisseur de matière à enlever est connue. Cette information nous permet de prédire avec un degré de certitude suffisant les efforts de coupe exercés par l'outil sur la pièce [19].

- L'entité de prise de pièce. Il s'agit du posage caractérisé par un type et un triplet d'appui. Chaque appui est caractérisé par la géométrie de la pièce participant à chaque appui. Le bridage est caractérisé par un mode de bridage et par un ensemble de lieux où il est possible de placer une bride type en évitant les collisions entre les outils de coupe et la bride. Cette information permet d'estimer l'existence d'une solution; en effet, les lieux de bridage ne correspondent pas aux lieux où seront placés les éléments technologiques de bridage. De plus, nous disposons des indicateurs de qualité, caractérisant l'espace dans lequel doit se situer la géométrie nominale de chaque appui, de stabilité caractérisant les risques de glissement et de basculement de la pièce sur ses appuis pendant l'usinage (Fig. 4).

À partir de ces informations nous avons pour objectif de :

- Sélectionner les éléments technologiques les mieux adaptés pour assurer les appuis et le bridage. Ces choix technologiques répondent aux contraintes de qualité d'accessibilité et de comportement mécanique.

- Placer ces éléments sur la pièce de manière à garantir la qualité, l'accessibilité des outils de coupe aux surfaces à usiner et l'accessibilité des éléments technologiques du montage d'usinage, la stabilité et la maîtrise des déformations de la pièce durant son usinage (Fig. 5).

- De relier ces éléments technologiques à la plaque de base en garantissant l'assemblage des éléments entre eux sans collision et la possibilité de monter et de démonter l'ensemble des pièces de la série tout en respectant les cadences et les conditions environnementales liées à la présence de copeau et de 


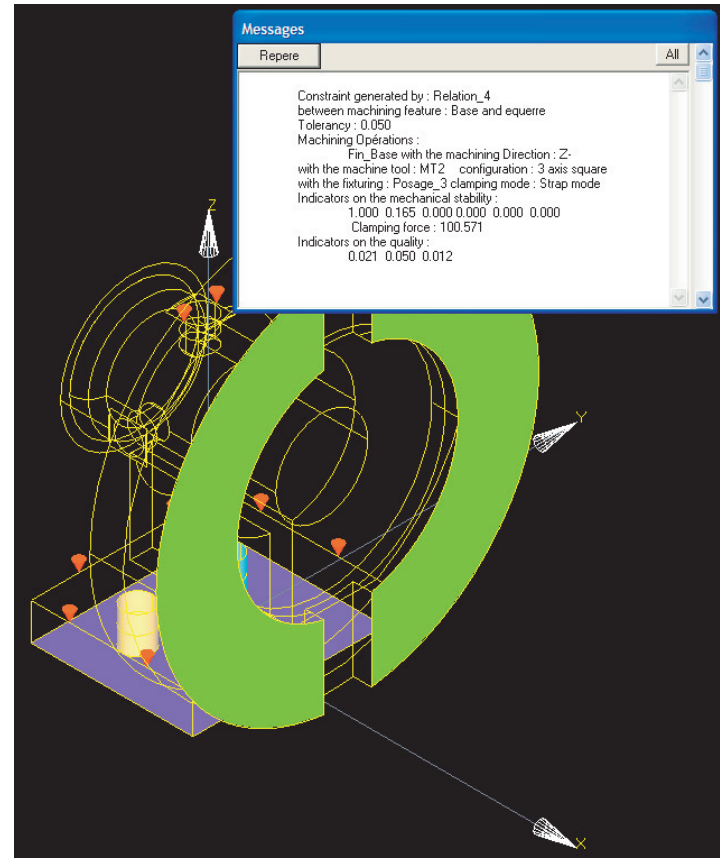

Fig. 4. Modèle de prise de pièce.

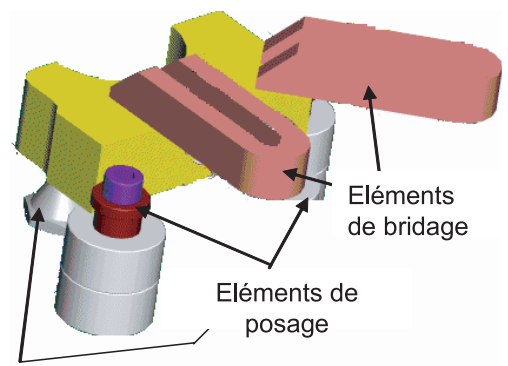

Fig. 5. Placement des éléments d'appui et de bridage sur la pièce.

fluide de coupe. De plus, le montage d'usinage doit être rigide et compact pour éviter les déformations excessives sous l'effet des efforts de coupe ainsi que toutes vibrations indésirables. L'évacuation des copeaux et du lubrifiant est importante pour assurer la propreté des contacts entre la pièce et le montage d'usinage (Fig. 6).

\section{Démarche de conception}

La démarche générale de conception du montage d'usinage modulaire pour le fraisage passe par trois étapes qui sont montrées dans l'organigramme de la figure 7 .

En entrées nous avons la cinématique de la machine outil, l'orientation de la pièce dans le repère de la machine-outil, la liste des opérations d'usinage à effectuer et l'entité de prise de pièce.

De plus nous disposons d'une base de données comportant l'ensemble des informations sur les éléments modulaires.

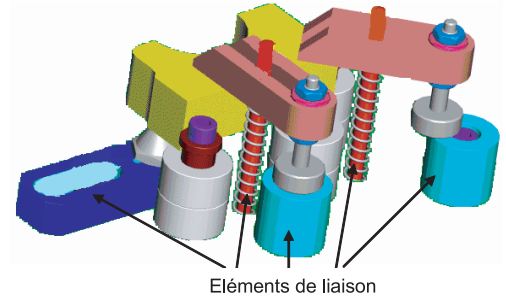

Fig. 6. Éléments de liaison entre les appuis, les brides et la plaque de base.

\begin{tabular}{|c|c|}
\hline $\begin{array}{c}\text { Entité de prise de pièce } \\
\text { Triplet d'appui } \\
\text { Mode de bridage } \\
\text { Indicateurs de performances }\end{array}$ & $\begin{array}{c}\text { Machine-outil } \\
\text { Cinématique } \\
\text { Configuration }\end{array}$ \\
\hline $\begin{array}{c}\text { Opérations d'usinage } \\
\text { Type d'opération } \\
\text { Outils de coupe }\end{array}$ & $\begin{array}{c}\text { Base de données } \\
\text { Éléments modulaires } \\
\text { Définition CAO des éléments }\end{array}$ \\
\hline
\end{tabular}

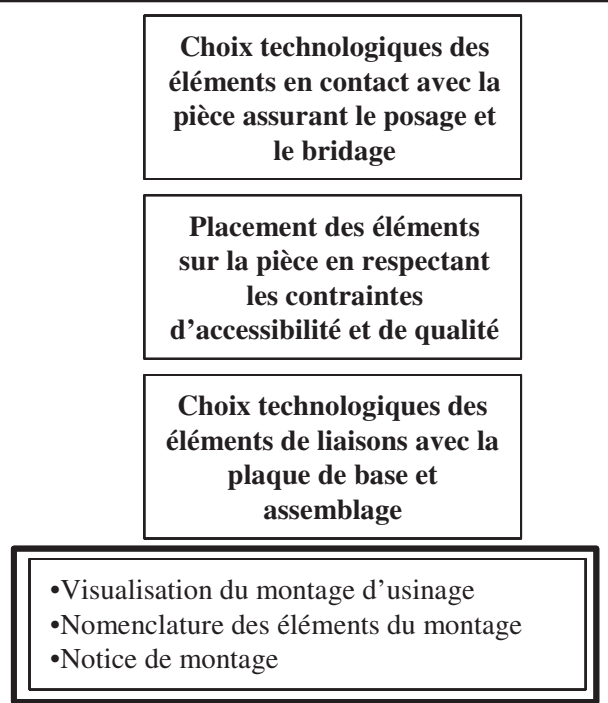

Fig. 7. Les différentes étapes de conception d'un montage d'usinage.

En sortie, nous désirons obtenir un montage d'usinage complètement conçu dans un environnement CFAO. De plus, nous souhaitons générer l'ensemble des informations concernant l'utilisation du montage d'usinage. Cette étape est actuellement en cours de développement.

\subsection{Choix technologiques des éléments en contact avec la pièce}

Durant cette étape, nous devons tout d'abord préciser comment cette mise en position doit être réalisée puis ensuite choisir les éléments technologiques devant assurer l'appui prépondérant puis les éléments technologiques devant assurer les appuis secondaires et tertiaires. Dans certains cas, il est judicieux d'utiliser des éléments assurant partiellement l'appui prépondérant et l'appui secondaire. 
Le choix technologique des éléments de bridage n'est pas complètement indépendant de la solution technologique des appuis. En effet, pour éviter les déformations, il est intéressant de brider en face des points d'appui. Les fournisseurs de montages modulaires proposent des éléments assurant l'appui et un bridage opposé à cet appui.

La définition d'éléments de soutien est activée lorsque le risque de déformation ou de vibration est important. Nous proposons de découpler le choix des éléments de soutien des choix des éléments d'appui et de bridage et traiter le problème de soutien après avoir choisi les éléments d'appui et de bridage.

Le choix des éléments technologiques est essentiellement issu de règles expertes. Ces règles sont formalisées pour répondre aux contraintes de qualité, d'accessibilité et de comportement mécanique. Ces choix sont essentiellement fonction :

- De l'état de la surface d'appui. Les appuis peuvent être réalisés sur une surface brute : moulée, forgée, laminée ou sur une surface usinée ou étirée. Pour respecter la qualité et éviter les déformations, il est préférable de localiser les points d'appui lorsque la surface est de mauvaise qualité. De plus lorsque l'appui est obtenu à l'aide de plusieurs géométries de la pièce, il est difficile de réaliser un appui sur toute l'étendue de la surface d'appui.

- Des caractéristiques mécaniques de la matière de la pièce. La pression de contact au droit des points d'appui doit rester compatible avec les caractéristiques mécaniques du matériau pour éviter des déformations permanentes. Ces déformations ne sont pas rédhibitoires si la surface d'appui est usinée par la suite. Par contre, les déformations devront dans tous les cas être compatibles avec la qualité d'appui demandée. Il est parfois intéressant d'améliorer le coefficient de frottement à l'aide de touches striées qui créent un obstacle au glissement par déformations plastiques de la pièce au droit de la touche striée.

- De la rigidité de la pièce. Le manque de rigidité de la pièce nous impose de coupler les choix technologiques pour le posage et le bridage. La solution technologique adoptée consiste à placer l'élément de bridage en face du point d'appui. De plus, des appuis rotulés garantissent un auto alignement de la surface d'appui appartenant à la pièce avec l'appui.

\subsection{Placement des éléments en contact avec la pièce}

Le placement des éléments du montage d'usinage est optimisé de manière à répondre aux contraintes de :

- Stabilité. Les éléments constituant l'appui prépondérant seront placés de manière à obtenir le triangle de sustentation ayant la plus grande étendue. De plus, cette stabilité est maximale si l'effort résultant de la coupe et du bridage passe au plus près du centre de gravité du triangle de sustentation formé par les trois points de l'appui prépondérant.

- Qualité. Le placement des points d'appui répond aussi à des contraintes issues de la qualité de la surface d'appui. Il est en effet déconseillé de se positionner sur les zones où les défauts de la pièce sont importants comme par exemple les bavures issues du plan de joint de fonderie ou de forge. Dans tous les cas, la géométrie de chaque appui devra rester dans l'espace défini par l'indicateur de qualité (Fig. 4).

- Accessibilité. Les éléments du montage d'usinage devront avoir accès aux surfaces de la pièce et ne devront pas entrer en collision entre eux, ni avec les outils de coupe et la pièce.

- Déformations. Les éléments du montage d'usinage sont placés de manière à minimiser les déformations de la pièce. Le couplage entre les éléments d'appui et de bridage est essentiel pour minimiser ces déformations. Il n'est donc pas possible de gérer de manière séquentielle la conception du posage puis du bridage. La stratégie retenue sur les pièces déformables est de placer les brides en face des éléments assurant les points d'appui.

Ces différentes contraintes ne sont pas indépendantes entre elles. Il est donc difficile de les satisfaire toutes de manière optimale. La solution retenue sera donc issue d'un compromis entre toutes ces contraintes. Pour cela, nous nous proposons de mettre en place une fonction optimisant le placement de ces éléments tout en respectant au mieux les contraintes.

Les efforts de coupe sont calculables pendant l'étude de chaque sous-phase. En effet ils sont dépendants de paramètres (profondeur de passe, avance, matériau de l'outil, ...) qui sont déterminés dans l'étude de la sousphase. Comme l'entité prise de pièce est indispensable en amont de la conception du montage d'usinage, nous avons donc mené une étude pour estimer les efforts de coupe par opération d'usinage [19]. Cette estimation est suffisante pour prendre les décisions lors de la conception du montage d'usinage. Cette approche nous permet de gérer en parallèle la conception des trajectoires d'usinage et la conception du montage d'usinage.

À partir de l'estimation des efforts de coupe, nous disposons de l'ensemble des informations pour concevoir et valider le montage d'usinage. Il est en effet possible de calculer l'incidence de ces efforts sur les points d'appui, valider la stabilité, les déformations locales et globales de la pièce.

\subsection{Conception du montage d'usinage modulaire}

Il s'agit maintenant de relier les éléments en contact avec la pièce à la plaque de base.

Les critères à respecter sont :

- Compacité. Pour limiter les dimensions du montage d'usinage et rester dans les courses de la machineoutil, il est souhaitable de placer la pièce au plus 
près de la plaque de base. Il est cependant nécessaire de penser aux risques de collision entre la broche ou l'outil de coupe et le montage d'usinage. De plus, les éléments ne peuvent pas être placés au droit des trous de la plaque de base. Il est donc nécessaire de laisser un espace suffisant pour les éléments assurant la liaison entre les éléments en contact avec la pièce et la plaque de base.

- Rigidité. Le montage d'usinage doit avoir une rigidité importante pour éviter les risques de vibration durant l'usinage de la pièce. Le critère de compacité répond aussi à cette contrainte.

- Aptitude à l'emploi. Durant son utilisation le montage d'usinage est dans un environnement fortement pollué par les copeaux et le fluide de coupe. La conception du montage d'usinage devra permettre une bonne évacuation de ces éléments de manière à éviter de perturber le fonctionnement du système d'échange de porte-pièce. De plus, durant le chargement et déchargement de la pièce les surfaces en contact avec la pièce doivent rester propres de manière à éviter une erreur de mise en position de la pièce dans le repère d'usinage.

\section{Conclusion}

Dans cet article, nous avons proposé une démarche de conception d'un montage d'usinage réalisé à l'aide d'éléments modulaires. Nous avons mis en évidence les trois grandes étapes; choix technologiques des éléments en contact avec la pièce puis placement de ces éléments en répondant au mieux aux différentes contraintes de qualité, d'accessibilité et de comportement mécanique et enfin la conception de l'ossature du montage répondant à des critères de compacité et de rigidité.

Maintenant, nous implémentons cette démarche dans un environnement CFAO Open CasCade, environnement déjà retenu pour l'identification et la reconnaissance des prises de pièce et la génération de gammes d'usinage.

\section{Références}

[1] E. Caillaud, Modélisation des connaissances : aide à la conception des montages d'usinage, Thèse, Université de Bordeaux I, 1995

[2] J.-L. Hou, A.J.C. Trappey, Computer-aided fixture design system for comprehensive modular fixtures, Int. J. Prod. Res. 39 (2001) 3703-3725

[3] J. Li, W. Ma, Y. Rong, Fixturing surface accessibility analysis for automated fixture design, Int. J. Prod. Res. 37 (1999) 2997-3016
[4] Z.J. Tao, A.S. Kumar, A.Y.C. Nee, A computational geometry approach to optimum clamping synthesis of machining fixtures, Int. J. Prod. Res. 37 (1999) 3495-3517

[5] W. Xie, S. Choudhuri, V. Subramanian, E.C. De Meter, M.W. Trethewey, Experimental assessment of a clamp actuation intensity analysis model, Int. J. Prod. Res. 38 (2000) 4611-4633

[6] A.Y.C. Nee, A.S. Kumar, Z.J. Tao, An intelligent fixture with a dynamic clamping scheme, Proc. Inst. Mech. Engrs. 214 (2000) 183-196

[7] B. Li, S.N. Melkote, Fixture clamping force optimisation and its impact on workpiece location accuracy, Int. J. Adv. Man. Tech. 17 (2001) 104-113

[8] J. Cecil, A Clamping Design Approach for Automated Fixture Design, Int. J. Adv. Man. Tech. 18 (2001) 784-789

[9] J. Cecil, Computer-Aided Fixture Design, A Review and Future Trends, Int. J. Adv. Man. Tech. 18 (2001) 790-793

[10] Y. Wu, Y. Rong, W. Ma, S.R. LeClair, Automated modular fixture planning: geometric analysis, Rob. Comp. Int. Man. 14 (1998) 1-15

[11] A.S. Kumar, V. Subramaniam, K.C. Seow, Conceptual Design of Fixtures using Genetic Algorithms, Int. J. Adv. Man. Tech. 15 (1999) 79-84

[12] Y. Rong, W. Hu, Y. Kang, Y. Zhang, D.W. Yen, Locating error analysis and tolerance assignment for computer-aided fixture design, Int. P. Prod. Res. 39 (2001) 3529-3545

[13] A.S. Kumar, J.Y.H. Fuh, T.S. Kow, An automated design and assembly of interference-free modular fixture setup, Computer-Aided Design 32 (2000) 583-596

[14] W. Hu, Y. Rong, A fast interference checking algorithm for automated fixture design verification, Int. J. Adv. Man. Tech. 16 (2000) 571-581

[15] Y.G. Liao, S.J. Hu, An integrated model of a fixtureworkpiece system for surface quality prediction, Int. J. Adv. Man. Tech. 17 (2001) 810-818

[16] M.Y. Wang, D.M. Pelinescu, Contact force prediction and force closure analysis of a fixtured rigid workpiece with friction, J. Man. Sc. and Eng. 125 (2003) 316-324

[17] H. Paris, D. Brissaud, Integration of the machining point of view in the product modelling : the fixuring features, in Integrated Design and Manufacturing in Mechanical Engineering, Kluwer Academic Publishers, ed. Chedmail, Bocquet, Dornfeld, 1997, ISBN 0-7923-4739-O, IDMME conference, Nantes, May 1996, pp. 121-130

[18] H. Paris, D. Brissaud, Process planning strategy based on fixturing indicators evaluation, Int. J. Adv. Man. Tech. 25 (2005) 913-922

[19] H. Paris, A. Voisin, Estimate of workpiece deformations while machining in a process planning Context, in 3rd International Conference on Integrated Design and Manufacturing in Mechanical Engineering, IDMME'2000, Montréal 17-19 May 2000, Presses Internationales Polytechnique, ed. C. Mascle, C. Fortin, J. Pegna, 2000 